\section{Deutschland droht europäisch festgelegtes Klimaziel für 2020 zu verfehlen}

Die klimapolitische Diskussion ist auf das deutsche Ziel ausgerichtet, die Emissionen bis 2020 um 40\% gegenüber 1990 zu reduzieren. Es gibt aber noch weitere, rechtlich verbindliche, Klimaziele für 2020 und seit Kurzem auch für 2030, auf die sich ein näherer Blick lohnt. Von Jan Nill

D as EU-Recht fordert in Sektoren, die nicht unter das EU-Emissionshandelssystem fallen, eine Verringerung der deutschen Treibhausgasemissionen um 14\% zwischen 2005 und 2020. Davon betroffen sind Wirtschaftszweige wie Verkehr, Gebäude, Landwirtschaft und Abfall. Diese sind immerhin für rund $50 \%$ der deutschen Gesamtemissionen verantwortlich.

Die Umsetzung dieses Ziels beinhaltet jährliche Emissionszuweisungen für 2013 bis 2020 (vgl. Abbildung 1), um die Zielerreichung sicherzustellen und die Anwendung von Flexibilitätsmechanismen zu ermöglichen. 2013 bis 2015 blieben die deutschen Emissionen unter den

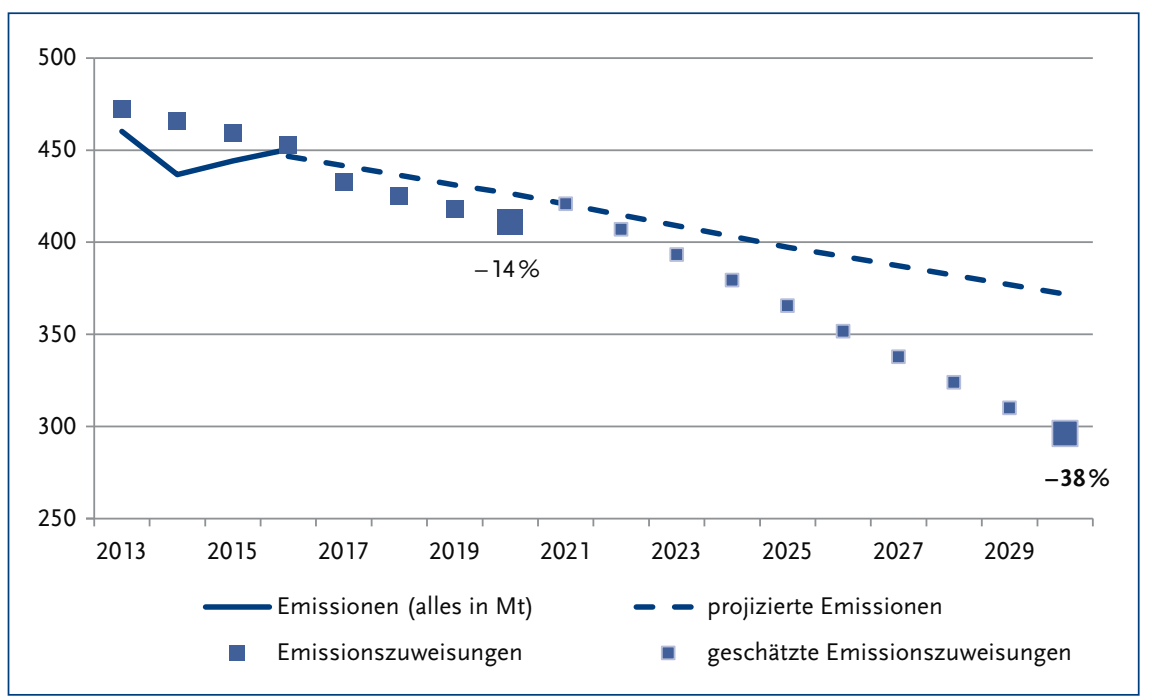

Abbildung 1: Emissionstrends und verbindliche Klimaziele

(Quelle: Autor, mit Daten aus Europäische Umweltagentur [2017]: Trends and Projections in Europe 2017) auf Flexibilitätsmechanismen zurückgreifen: zum Beispiel den Übertrag der bisher erzielten Überschüsse und den Ankauf von Emissionszuweisungen anderer EU-Mitgliedsstaaten.

Inzwischen ist die Europäische Gesetzgebung aber schon weiter. Kürzlich wurden die EU-Klimaschutzgesetze für die Jahre 2021-30 beschlossen. Diese regeln, wie das EU-Klimaziel für 2030 von $40 \%$ Emissionsminderungen im Vergleich zu 1990 umgesetzt wird. Für Deutschland legt dies für Sektoren, die nicht unter das EU-Emissionshandelssystem fallen, ein Ziel von 38\% weniger Emissionen als in 2005 fest. Vergleicht man dies mit den aktuellen deutschen Emissionsprojektionen, so ist eine erhebliche Lücke zu schließen, die weit grösser als die Lücke bis 2020 ist (vgl. Abbildung 1). Wie bisher werden jährliche verbindliche Emissionsminderungsziele 2021 bis 2030 gesetzt, wobei der Beginn der Trajektorie von den realen Emissionen 2016 bis 2018 abhängen wird. Etwaige Überschüsse aus der Periode 2013 bis 2020 können nicht übertragen werden. Aber es bestehen weiterhin Flexibilitätsmechanismen, die eine kosteneffiziente Zielerreichung ermöglichen.

In erster Linie ist es an den Mitgliedstaaten, darüber zu entscheiden, mit welchen Maßnahmen das Ziel für 2030 erreicht werden kann. Integrierte nationale Energie und Klimapläne, für die das EU-Gesetzgebungsverfahren kurz vor dem Abschluss steht, können hierfür ein wichtiges Instrument sein. Spezifische europäische Politiken wie $\mathrm{CO}_{2}$-Standards für Fahrzeuge können ebenfalls dazu beitragen, es wird interessant sein zu sehen, welche Position Deutschland zu den dafür von der Kommission vorgelegten Gesetzesvorschlägen einnimmt.

AUTOR + KONTAKT

Dr.Jan Nill ist Referent für Klimapolitik in Nicht-Emissionshandels-Sektoren in der Europäischen Kommission.

Europäische Kommission, Generaldirektion Klimapolitik, Referat KLIMA.C.2. Tel.: +32 22990190 E-Mail: Jan.Nill@ec.europa.eu and redistributing the material in any medium or format, provided the original work is properly cited, it is not used for commercial purposes and it is not remixed, transformed or built upon. The access to the digital version of this article is reserved to subscribers of ÖklogischesWirtschaften until two years after the date of publication; after two years it is available to all readers. 